\title{
Co-products in maize-soybean growing-pig diets altered in vitro enzymatic insoluble fibre hydrolysis and fermentation in relation to botanical origin
}

\author{
F. Fushai ${ }^{1 \#}$, M. Tekere ${ }^{2}$, M. Masafu ${ }^{2}$, C.M Akinsola ${ }^{3}$, F. Siebrits ${ }^{3}$, F.V. Nherera-Chokuda ${ }^{4}$ \\ \& A.T. Kanengoni ${ }^{5}$ \\ ${ }^{1}$ University of Venda, Private Bag X5050, Thohoyandou, Limpopo, 0950, South Africa \\ ${ }^{2}$ University of South Africa, P O Box 392, Florida 0003, South Africa \\ ${ }^{3}$ Tshwane University of Technology, Private Bag X680, Pretoria 0001, South Africa \\ ${ }^{4}$ ARC-Animal Production Institute, P/Bag X2, Irene, 0062, South Africa \\ ${ }^{5}$ Johannesburg Zoo, Private Bag X13, Parkview, 2122, South Africa
}

(Received 20 June 2018; Accepted 17 December 2018; First published online 2 April 2019)

\author{
Copyright resides with the authors in terms of the Creative Commons Attribution 4.0 South African Licence. \\ See: http://creativecommons.org/licenses/by/4.0/za \\ Condition of use: The user may copy, distribute, transmit and adapt the work, but must recognise the authors and \\ the South African J. Anim. Sci.
}

\begin{abstract}
The study examined the effects of botanical factors and fermentation-based, high-level dilution of coproduct feeds in maize-soybean growing-pig diets on enzymatic insoluble fibre hydrolysis and fermentation. Feed insoluble fibre residues that were recovered after pepsin-pancreatin digestion were subjected to Roxazyme ${ }^{\circledR} \mathrm{G} 2$ (Roxazyme) versus Viscozyme $L^{\circledR}$ V2010 (control) hydrolysis, and to 64-hour fermentation using pig faecal inoculum. The control diet was a $13 \mathrm{MJ}$ metabolizable energy, $141 \mathrm{~g}$ total dietary fibre/kg dry matter maize-meal/hominy chop-soybean diet, which was diluted with maize cob, soybean hulls, barley brewer's grains, lucerne hay or wheat bran in $12 \mathrm{MJ}$ metabolizable energy, $246 \mathrm{~g}$ total dietary fibre/kg dry matter iso-nutrient, single co-product test diets. Fermentable insoluble fibre was employed in a computerized iterative selection of ingredients in two iso-nutrient $11 \mathrm{MJ}$ metabolizable energy 319 total dietary fibre/kg dry matter mixed fibre test diets for maximal contrast (high (HF) versus low (LF)) in fermentability. Insoluble fibre extractive pepsin-pancreatin digestibility differed between feed ingredients, and the single co-product test diets, and between the HF and LF mixed co-product diets. Fibre digestibility depended on both the origin and enzyme, with interaction, whereby carbohydrases expressed similar low $(0.04-0.05)$ insoluble fibre digestibility for maize cob, moderate (0.12) digestibility for wheat bran and brewer's grain, with inferior Roxazyme G2 digestibility for maize hominy chop (0.02 vs 0.10$)$ and meal $(0.04$ vs 0.16$)$, dehulled soybean meal (0.02 vs 0.17$)$, lucerne hay (0.08 vs 0.18$)$, and soybean hulls $(0.05$ vs 0.33$)$. Co-product-enzyme affinities were expressed in single fibre diets. Low Roxazyme-basal fibre affinity limited its comparative single co-product $(0.03-0.07$ vs $0.16-0.22)$ HF (0.07 vs 0.17$)$ and LF (0.4 vs 0.20$)$ dietary fibre digestibility. Screening for HF/LF did not affect enzymatic digestion, though enzyme combination increased HF, but not LF digestibility. Gas and short chain fatty acid production predicted fermentability proportionately in the declining order of dehulled soybean $\geq$ maize $\geq$ soy hulls $\geq$ maize hominy chop $>$ wheat bran >lucerne hay $\geq$ brewer's grain = maize cob. Induced HF and LF contrast was significant. Co-product fibre enrichment decreased fermentability for all except the soy hull and HF diets. Cereal fibre yielded proportionately less acetate, with more propionate and butyrate, and a greater butyrate shift for maize fibre. The HF fibre induced more ACE and less butyrate. Biomarkers of deleterious proteolytic fermentation were high for lucerne (isobutyrate) and soy hulls (iso-valerate). In conclusion, high-level and fermentation based co-product feed dilution into maize-soybean growing pig diets altered enzymatic insoluble fibre hydrolysis and fermentation in relation to botanical origin. Roxazyme expressed weak hydrolytic potency on maize and soybean insoluble fibre.
\end{abstract}

\footnotetext{
Keywords: fermentation gas, fermentation kinetics, fibre fermentability, insoluble non-starch polysaccharides, non-starch polysaccharide degrading enzymes, proteolytic fermentation, short-chain fatty acids

\#Corresponding author: felix.fushai@univen.ac.za
} 


\section{Introduction}

The processing of food grains into flour, vegetable oil, alcohol and biofuels offloads quantitatively variable, chemically diverse non-starch polysaccharides (NSP) into co-products (Bach Knudsen, 2014). Rapidly increasing human demand and random adverse climate change-related events are disrupting global grain markets, forcing producers into greater dependency on co-products for sustainable, cost-effective pig feeding strategies. Chemical and quantitative heterogeneity in co-product fibre and the consequent variable impact on nutrient extraction (Bach Knudsen, 2014; Swiatkiewicz et al., 2016) disturb established precisionfeeding systems, imposing risks on animal productivity and the environmental footprint (Zijlstra \& Beltranena, 2013; Woyengo et al., 2014).

Fitting co-products into efficient modern pig feeding systems requires effective evaluative methods to facilitate processing, and selective and calibrated dietary inclusion for optimal biological, economic and environmental outcomes. In growing pigs, strategic co-product feeding primarily involves targeting maximal fermentable fibre (Anguita et al., 2006; Gutierrez et al., 2013; lyayi \& Odeola, 2015) to offset fibre-induced nutrient and digestive metabolic energy wastage (Noblet \& Le Goff., 2001). Emerging evidence supports the consideration of dietary polysaccharides that promote gut health (Aumiller et al., 2015; Agyekum \& Nyachoti, 2017; Celi et al., 2018). Prebiotic fibre includes resistant starch, galacto and fructo oligosaccharides, mixedlinked $\beta$-glucans and soluble arabinoxylans (Aumiller et al., 2015). Beyond prebiosis, saccharolytic fermentation produces short chain fatty acids (SCFA), which beneficially modulate epithelial cell, and peripheral tissue metabolism (Byrne et al., 2015; Pieper et al., 2016). Butyrate (BUT) is the most enterotrophic and primary colonocyte energy substrate (Hamer et al., 2008; Tonel et al., 2010). In the intestinal epithelium, SCFA modulate nutrient transporter gene expression (Daly \& Shirazi-Beechey, 2006) digestive enzyme secretion (Mangian \& Tappenden, 2009) and mucosal immune homeostasis (Pieper et al., 2016). Peripherally, SCFA modulate satiety (Sleeth et al., 2010; Byrne et al., 2015), glucose (Theil et al., 2011) and lipid (Fushimi et al., 2006) metabolism to increase intermediary energy harvesting (Byrne et al., 2015). Saccharolysis spares proteolytic fermentation, which tends to intensify with depletion of fermentable NSP distal to the colon (Yao et al., 2016), thereby reducing ammonia (Zervas \& Zijlstra, 2002; Nahm, 2003), odours (Rideout et al., 2004) and toxic metabolites (Bach Knudsen, 2015). Biomarkers of deleterious proteolytic fermentation are the branched chain fatty acids (BCFA) iso-BUT, 2-methyl-butyrate and isovalerate (Ríos-Covián, et al., 2016; Celi et al., 2018), the metabolites of branched-chain amino acids, valine, leucine and isoleucine (Verbeke et al., 2015; Pieper et al., 2016), respectively.

Beneficial fermentation is a concept conceived and still largely confined to studies on temperate cereal test diets that are uniquely endowed with native or purified readily fermentable NSP (Bach Knudsen, 2014). However, the merging evidence increasingly links beneficial microbiota (Bifidobacterium and Lactobacilli spp) and fermentation patterns to a broader spectrum of fibre types (e.g. reviews by Molist et al., 2014; Aumiller et al., 2015; Jha \& Berrocoso, 2016; Simpson \& Campbell, 2015), which might include fermentable insoluble NSP (iNSP) in maize and all secondary plant tissues (Bach Knudsen, 2015). Among feeds, the fermentation of iNSP occurs in complex fashion, being subject to the primary and secondary structures and lignification, attributes which depend on the species, cultivar and morphological origin (Bach Knudsen, 1997; 2014), and are subject to environmental influences (Collins et al., 2010; De Vries et al., 2012; Aumiller et al., 2015). Whereas hydrolytic and fermentative degradation rapidly deplete the soluble NSP within the upper digestive tract (Noblet \& Le Goff, 2001; Back Knudsen, 2014), the iNSP are degraded to lesser and highly variable extents, mostly at the distal colon (Bach Knudsen, 1997; Noblet \& Le Goff, 2001). In monogastrics, exogenous polysaccharide degrading enzymes potentially improve the nutritional efficacy of complexly fibrous diets through substrate-dependent iNSP depolymerization to randomly polydisperse oligosaccharides (Choct et al., 2006). However, in pigs, current enzymes seem impotent, particularly on maize-soybean diets (Willamil et al., 2012; Agyekum \& Nyachoti, 2017), more so when the diets contain substantial co-product fibre (Kerr \& Shurson, 2013).

Fibre-gut microbe-pig interactions are complex (Zijilstra et al., 2010; Pieper et al., 2016). The best insight into the underpinning mechanics might be through a modelling approach (Zijilstra et al., 2010). Modelling in turn requires potent methods to mimic porcine fibre digestion for robust prediction of the biogenic effects (Lee at al., 2018), and to match enzymes to dietary NSP (Park et al., 2016a). Effective methods should be standardised, low cost, rapid and broadly applicable across a range of substrates. Pig fibre nutrition research employs a three-step (gastric-ileal-colon) in vitro hydrolytic digestive procedure (Boisen \& Fernandez, 1997). Mature sow rectal faecal microbiota inoculum are employed in batch fermentation of washed fibrous extracts from pepsin (gastric) + pancreatin (small intestinal) feed digestion (Bindelle et al., 2007), from which total SCFA and cumulative gas jointly predict insoluble fibre fermentability (Bindelle et al., 2007; Jonathan et al., 2012). Endpoint SCFA and microbiota (Verbeke et al., 2015; Agyekum \& Nyachoti, 2017) and the gas production kinetics (France et al., 1993; Groot et al., 1996) provide important 
insights into gut functionality. With wider testing, the approach might provide a practical evaluative tool for optimal co-product feeding.

The present study therefore employed the in vitro porcine digestion model to evaluate how the botanical origin, high-level and fermentation-based co-product dilution into maize-soybean soybean diets influence exogenous enzymatic and fermentative fibre degradation.

\section{Materials and Methods}

The procedures used for feed analyses, and enzymatic and fermentative digestion were approved by the Ethics Committees of the University of South Africa (Ref: 20111/CAES/024R1) and the Agricultural Research Council of South Africa (APIEC10/20).

Test feeds (Table 1) included maize (Zea mays), its hominy chop and cob, dehulled soybean (Glycine max), and the hulls, brewer's barley (Hordeum vulgare L) grains, lucerne (Medicago sativa) hay, and wheat (Triticum aestivum) bran. Representative feeds were sampled from coarse-milled bulk factory commercial feedstocks at OPTI Feeds (Pty) Ltd, Lichtenburg, South Africa, which were composites of co-product feeds sourced from grain produced in various agronomic systems. Feeds were milled through a 1-mm screen in an Ika analytical mill and oven-dried for 18 hours in a forced draught oven at $100{ }^{\circ} \mathrm{C}$.

A standard maize soybean mix (control) and two sets of test diets were formulated, in which treatments differed in the fibre types (Table 2a, 2b), but were equally balanced for essential nutrients according to the NRC (1998). Metabolizable energy was quantified from chemical components (Noblet \& Perez, 1993) (equation 30 and 43). In single co-product test diets, fibrous co-product feeds were singly incorporated into a standard (control) maize grain-hominy chop-soybean growing pig diet. In a second set of mixed co-product diets, the ingredients were screened within least cost diet formulation based on fermentable fibre content (g pepsin-pancreatin digesta/kg dry matter feed $\mathrm{x} \mathrm{mL}$ fermentation gas/kg dry matter of digesta) to maximize contrast (high (HF) versus low (LF)) in fibre fermentability.

Feeds were analysed using AOAC (2006) methods for dry matter (934.01), crude protein (990.03), ether extract (942.05), ash (920.39) and total dietary fibre (991.43). Neutral and acid detergent fibre were analysed according to Van Soest et al. (1991). Gross energy (GE) was determined by combustion in a DDS isothermal CP500 bomb calorimeter.

Feeds were enzymatically digested in a three-step porcine digestion model (Boisen \& Fernandez, 1997), with modifications for maximal digestion in an ANKOM Daisy ${ }^{\prime \prime}$ incubator. The incubator setup consisted of four digestion chambers, in one of which, for each enzyme digestive step, samples were sequentially incubated in separate batches of feed ingredients [( 3 replicates $\times 8$ feeds) +1 blank (no sample)], single [4 replicates $x(5$ test diets +1 control $)+1$ blank] and mixed co-product diets (12 replicates $x$ $\left(\mathrm{HF}+\mathrm{LF}\right.$ test diets) +1 blank]. The $39 \pm 0.5^{\circ} \mathrm{C}$ digestion of $0.5 \pm 0.01 \mathrm{~g}$ dry matter ground feed samples in $99 \%$ acetone-washed ANKOM ${ }^{\circledR}$ F57 filter bags was performed as follows.

Pepsin digestion: 2-hour incubation in $[600 \mathrm{~mL} 0.1 \mathrm{M}, \mathrm{pH} 6.0$ phosphate buffer $+240 \mathrm{~mL} 0.2 \mathrm{M} \mathrm{HCl}$ (adjusted to $2.0 \mathrm{pH}$ using $1 \mathrm{M}$ solutions of $\mathrm{HCl}$ or $\mathrm{NaOH}$ )] $+0.6 \mathrm{~g}$ fresh pepsin solution (porcine, $2000 \mathrm{FIP}$ $\mathrm{U} / \mathrm{g}$, Merck no. 7190) + $12 \mathrm{~mL}$ of chloramphenicol (Sigma no. C-0378, $0.5 \mathrm{~g} / 100 \mathrm{~mL}$ ethanol).

Pancreatin digestion: 5-hour incubation after topping up step 1 digestion medium with [240 $\mathrm{mL}$ of phosphate buffer $(0.2 \mathrm{M}, \mathrm{pH} 6.8)+120 \mathrm{~mL} 0.6 \mathrm{M} \mathrm{NaOH})(\mathrm{pH}$ adjusted to 6.8 using $1 \mathrm{M} \mathrm{HCl} \mathrm{or} \mathrm{NaOH})]+2.4$ $\mathrm{g}$ pancreatin (porcine, grade IV, Sigma no. P-1750).

Fibrolysis: 24-hour incubation in $750 \mathrm{~mL}$ fresh phosphate buffer $(0.1 \mathrm{M}, \mathrm{pH} 4.8)+$ either $12 \mathrm{~mL}$ Viscozyme (Viscozyme $\mathrm{L}^{\circledR}$ V2010 $120 \mathrm{~L}$, mixture including $\beta$-glucanase, xylanase, arabinase, cellulase (120 FBG/g)), Novo Nordisk, Bagsvaerd), or 0.17 g Roxazyme (Roxazyme ${ }^{\circledR}$ G2 DSM Pvt Ltd, with endo-1,4- $\beta$ glucanase (8000 U/g), endo-1,3 (4)- $\beta$-glucanase (18000 U/g) and endo-1,3 (4)- $\beta$-xylanase (26000 U/g). For HF and LF fibrous residues, the cocktails were mixed in a third treatment to test synergistic/complementary enzyme effects.

Dry matter lost after the second and third digestion steps was estimated gravimetrically by washing off fat and soluble residue through sequential gentle rinsing in warm tap water, 95\% ethanol and 99\% acetone, before forced air oven-drying at $85^{\circ} \mathrm{C}$ for 18 hours. 
Table 1 Variation in dry matter $(\mathrm{g} / \mathrm{kg})$, chemical ( $\mathrm{g} / \mathrm{kg}$ dry matter) and energy (MJ/kg dry matter) composition among test pig feed ingredients of diverse botanical origin

\begin{tabular}{|c|c|c|c|c|c|c|c|c|}
\hline \multirow[b]{2}{*}{ Components } & \multicolumn{8}{|c|}{ Feed ingredient } \\
\hline & Maize graiı & $\begin{array}{l}\text { Maize hominy } \\
\text { chop }\end{array}$ & Maize cobs & $\begin{array}{l}\text { Brewer's } \\
\text { grains }\end{array}$ & $\begin{array}{l}\text { Wheat } \\
\text { bran }\end{array}$ & Lucerne hay & $\begin{array}{l}\text { Dehulled } \\
\text { soybean }\end{array}$ & Soybean hulls \\
\hline Dry matter & 901 & 896 & 944 & 875 & 902 & 946 & 904 & 913 \\
\hline Organic matter & 989 & 979 & 944 & 956 & 951 & 910 & 929 & 951 \\
\hline Gross energy & 16 & 18 & 17 & 22 & 17 & 16 & 18 & 16 \\
\hline Crude protein & 62 & 97 & 42 & 241 & 167 & 161 & 515 & 119 \\
\hline Ether extract & 36 & 71 & 11 & 73 & 32 & 17 & 13 & 11 \\
\hline Crude fibre & 13 & 59 & 352 & 212 & 90 & 381 & 43 & 421 \\
\hline Neutral detergent fibre & 121 & 331 & 811 & 752 & 443 & 518 & 148 & 703 \\
\hline Acid detergent fibre & 29 & 103 & 669 & 280 & 131 & 454 & 66 & 542 \\
\hline Hemi-cellulose ${ }^{1}$ & 92 & 228 & 142 & 472 & 312 & 64 & 82 & 161 \\
\hline Acid detergent lignin & 20 & 18 & 47 & 57 & 41 & 94 & 13 & 36 \\
\hline Cellulose $^{2}$ & 9 & 85 & 622 & 223 & 90 & 360 & 53 & 506 \\
\hline Soluble dietary fibre & 4 & 9 & 5 & 18 & 17 & 26 & 7 & 14 \\
\hline Insoluble dietary fibre & 113 & 249 & 871 & 627 & 363 & 600 & 152 & 773 \\
\hline Total dietary fibre & 118 & 258 & 876 & 646 & 380 & 627 & 158 & 787 \\
\hline
\end{tabular}

${ }^{1}$ Neutral detergent fibre - acid detergent fibre
${ }^{2}$ Acid detergent fibre - acid detergent lignin 
Table 2a Feed ingredient $(\mathrm{g} / \mathrm{kg})$ composition of experimental growing pig diets

\begin{tabular}{|c|c|c|c|c|c|c|c|c|}
\hline \multirow{3}{*}{ Ingredients } & \multirow{3}{*}{ Standard } & \multicolumn{7}{|c|}{ Diets } \\
\hline & & \multicolumn{7}{|c|}{ Added dietary insoluble fibre type } \\
\hline & & Soybean hulls & Maize cobs & Wheat bran & Lucerne hay & Brewer's grains & Low fermentability & High fermentability \\
\hline Maize & 560 & 390 & 394 & 209 & 383 & 401 & 273 & 282 \\
\hline Maize hominy chop & 65 & 65 & 65 & 65 & 65 & 65 & 65 & 65 \\
\hline Maize cob & - & & 142 & & & & 108 & 57 \\
\hline Dehulled soybean & 300 & 329 & 350 & 262 & 303 & 267 & 280 & 322 \\
\hline Soybean hulls & & 169 & & & & & & 203 \\
\hline Brewer's grains & & & & & & 219 & 202 & \\
\hline Wheat bran & 25 & & & 423 & & & 25 & 25 \\
\hline Lucerne hay & & & & & 204 & & & \\
\hline DL methionine & 2 & 2 & 2 & 2 & 2 & 2 & 2 & 2 \\
\hline Limestone flour & 26 & 23 & 24 & 24 & 21 & 25 & 25 & 22 \\
\hline L-lysine $\mathrm{HCl}$ & 1 & - & & & & & & \\
\hline Monocalcium phosphate & 11 & 11 & 11 & 3 & 11 & 10 & 10 & 1.1 \\
\hline Sodium chloride & 10 & 10 & 10 & 10 & 10 & 10 & 10 & 10 \\
\hline Vitamin \& mineral premix & 2 & 2 & 2 & 2 & 2 & 2 & 2 & 2 \\
\hline
\end{tabular}


Table $\mathbf{2 b}$ Chemical (g/kg dry matter) and energy (MJ/kg dry matter) composition of experimental growing pig diets

\begin{tabular}{|c|c|c|c|c|c|c|c|c|}
\hline \multirow{3}{*}{ Components } & \multicolumn{8}{|c|}{ Diets } \\
\hline & \multirow{2}{*}{ Standard } & \multicolumn{7}{|c|}{ Added dietary insoluble fibre type } \\
\hline & & Soybean hulls & Maize cobs & Wheat bran & Lucerne hay & Brewer's grains & Low fermentability & High fermentability \\
\hline Dry matter & 897 & 900 & 904 & 902 & 901 & 906 & 926 & 921 \\
\hline Organic matter & 922 & 907 & 912 & 921 & 905 & 919 & 921 & 918 \\
\hline Ether extract & 30 & 25 & 25 & 29 & 26 & 38 & 32 & 22 \\
\hline Crude protein & 219 & 233 & 229 & 233 & 233 & 232 & 227 & 226 \\
\hline Starch & & & & & & & 286 & 278 \\
\hline Gross energy & 15 & 16 & 17 & 17 & 16 & 15 & 17.7 & 17.1 \\
\hline Metabolizable energy & 13 & 12 & 12 & 12 & 12 & 12 & 11 & 11 \\
\hline Neutral detergent fibre & 146 & 239 & 242 & 274 & 224 & 269 & 337 & 331 \\
\hline Acid detergent fibre & 47 & 133 & 141 & 86 & 135 & 96 & 163 & 187 \\
\hline Hemicellulose $^{1}$ & 99 & 106 & 101 & 188 & 89 & 173 & 174 & 144 \\
\hline Acid detergent lignin & 17 & 20 & 21 & 26 & 33 & 25 & 27 & 22 \\
\hline Cellulose $^{2}$ & 30 & 113 & 120 & 60 & 102 & 71 & 310 & 309 \\
\hline Soluble dietary fibre & 6 & 7 & 5 & 10 & 10 & 8 & 8 & 7 \\
\hline Insoluble dietary fibre & 135 & 243 & 243 & 234 & 234 & 235 & 312 & 310 \\
\hline Total dietary fibre & 141 & 250 & 249 & 244 & 244 & 243 & 320 & 318 \\
\hline
\end{tabular}

${ }^{1}$ Neutral detergent fibre - acid detergent fibre

${ }^{2}$ Acid detergent fibre - acid detergent lignin 
Washed fibre isolates were fermented in pig faecal inoculum, according to Bindelle et al. (2007), with modifications for optimum fermentation in an ANKOM ${ }^{R F}$ automated gas measurement system. The setup had $10 \times 200 \mathrm{~mL}$ fermentation modules, in which substrates were fermented separately in different runs, namely [2 blanks (no substrate) +8 feed ingredients], [2 blanks +1 control +5 single fibre diets], 2 [1 blank +1 control $+1 \mathrm{HF}+1 \mathrm{HF}$ mixed fibre diets]. The blank contained no substrate to estimate particle attachment. Fermentation was optimized by standard $39{ }^{\circ} \mathrm{C}$ anaerobic procedures. manual.

Buffer: Marten \& Barnes (1980) phosphate buffer, according to the Ankom Gas production system

Inoculum: Pooled rectal faecal aliquots from three healthy mature Large White-Landrace crossbred sows on a standard antibiotic-free dry sow ration, homogenized in batches of $60 \mathrm{~g}$ in $400 \mathrm{~mL}$ buffer by 60-second stomacher pummelling, filtered through four layers of mutton cloth.

Fermentation: $0.75 \mathrm{~g} \pm 0.001 \mathrm{~g}$ dry matter substrate $+100 \mathrm{~mL}$ buffer $+50 \mathrm{~mL}$ inoculum, 64-hour cumulative SCFA and gas production, gas measured by pressure-volume transduction at five-minute intervals.

Fermentation was terminated by immersing the modules in iced water for 15 minutes. Representative fermentation residues were transferred into airtight vials, and frozen-stored at $-20{ }^{\circ} \mathrm{C}$. To determine the SCFA and BCFA, frozen residues were thawed and purified by passing through Cameo 30 (0.45 $\mu \mathrm{m})$ filters. Aliquots of the filtrate were injected into a Varian 3300 FID detector gas chromatograph using a CP Wax 58 (FFAP) CB Cat no 7654 column $(25 \mathrm{~m}, 0.53 \mathrm{~mm}, 2.0 \mu \mathrm{m})$ in which helium was the carrier gas. The column temperature programme started at $50{ }^{\circ} \mathrm{C}$ for 2 minutes, increasing at $15{ }^{\circ} \mathrm{C}$ per minute to a constant final temperature of $190^{\circ} \mathrm{C}$ for 5 minutes.

The kinetics of fermentation gas production were described by fitting a nonlinear, monophasic algorithm (Groot et al., 1996) using the general linear models of SAS (2010):

where: $G V$ : gas volume (mL/g dry matter) at time $\mathrm{T}$

$$
\mathrm{GV}=\frac{A}{\left(1+\frac{T^{1 / 2}}{T}\right)^{2}}
$$

A: gas volume ( $\mathrm{mL} / \mathrm{g}$ dry matter) at $\mathrm{T}=\infty$

$\mathrm{T}$ : time (hours)

$\mathrm{T}_{1 / 2}$ : time (hours), when $\mathrm{GV}=\mathrm{A} / 2$

Factorial ANOVA of fermentation parameters (Model I) and enzymatic digestibility coefficients (Model II) were performed using the PROC MIXED procedures of SAS (2010):

$$
\begin{aligned}
& \mathrm{Y}_{i}=\mu+\alpha_{i}+\mathrm{e}_{i} \\
& \mathrm{Y}_{i j}=\alpha_{i}+\beta_{j}+(\alpha \beta)_{i j}+e_{i j}
\end{aligned}
$$

Model I

Model II

where: $\mu$ defined the overall mean

$\alpha_{i}$ the effect of the fibre type

$\beta_{\mathrm{j}}$ the enzyme effect

$(\alpha \beta)_{i j}$ the fibre-enzyme interactions

$\mathrm{i}$ and $\mathrm{j}$ the respective treatment levels

$\mathrm{e}_{\mathrm{i}}$, and $\mathrm{e}_{\mathrm{ij}}$, the random errors

Treatment means were separated using the Bonferroni $t$-test at $P=0.05$.

\section{Results}

Feed pepsin-pancreatin digestibility (Table 3) differed among feed ingredients $(P<0.001)$, the single co-product test diets $(P<0.0001)$, and the HF and LF mixed co-product diets $(P<0.001)$. Partial multienzymatic insoluble fibre digestibility (Table 4$)$ depended on both the origin $(P<0.001)$ and the enzyme $(P<0.001)$, with interaction $(P<0.001)$, whereby the enzymes had similarly $(P>0.05)$ low digestibility of maize cob fibre and moderate digestibility of wheat bran and brewer's grain, with inferior $(P<0.05)$ Roxazyme digestibility of maize hominy chop, maize meal, dehulled soybean meal, lucerne hay, and soybean hull fibre. The pattern of enzyme-feed fibre affinities was expressed in the single fibre co-product diets. Inferior $(P<0.05)$ Roxazyme basal fibre digestibility limited $(P<0.05)$ the digestibility of both the single and mixed co-product-maize-soybean dietary fibre. While the HF/LF feed screening resulted in similar 
enzymatic digestibility, the combination of the enzyme products increased $(P<05)$ the digestion of $\mathrm{HF}$, but not of LF fibre.

Table 3 Pepsin-pancreatin feed digestibility for insoluble residue extraction $(n=36)$

\begin{tabular}{|c|c|c|c|}
\hline & Mean & SEM & $P$-values \\
\hline \multicolumn{4}{|l|}{ Feed ingredients } \\
\hline Brewer's grains & $0.31^{f}$ & \multirow{8}{*}{0.014} & \multirow{8}{*}{$<0.001$} \\
\hline Maize cobs & $0.16^{\mathrm{g}}$ & & \\
\hline Maize hominy chop & $0.71^{\mathrm{c}}$ & & \\
\hline Maize & $0.75^{\mathrm{b}}$ & & \\
\hline Dehulled soybean meal & $0.80^{\mathrm{a}}$ & & \\
\hline Lucerne hay & $0.35^{\mathrm{e}}$ & & \\
\hline Soybean hulls & $0.29^{f}$ & & \\
\hline Wheat bran & $0.58^{\mathrm{d}}$ & & \\
\hline \multicolumn{4}{|c|}{ Standard $^{1}$ and single ${ }^{2}$ fibre diets } \\
\hline Standard & $0.70^{\mathrm{a}}$ & \multirow{6}{*}{0.003} & \multirow{6}{*}{$<0.0001$} \\
\hline Brewer's grains & $0.64^{\mathrm{c}}$ & & \\
\hline Maize cobs & $0.68^{\mathrm{b}}$ & & \\
\hline Lucerne hay & $0.67^{\mathrm{bc}}$ & & \\
\hline Soybean hulls & $0.65^{\mathrm{c}}$ & & \\
\hline Wheat bran & $0.68^{\mathrm{b}}$ & & \\
\hline \multicolumn{4}{|l|}{ Mixed fibre diets ${ }^{3}$} \\
\hline Low fermentability & $0.55^{\mathrm{b}}$ & \multirow{2}{*}{0.005} & \multirow{2}{*}{$<0.001$} \\
\hline High fermentability & $0.60^{\mathrm{a}}$ & & \\
\hline
\end{tabular}

abcdf For each set of means, values that do not share a common superscript are different at $P<0.05$

SEM: Standard error of the mean

${ }_{1}^{1} 13 \mathrm{MJ}$ metabolisable energy, $141 \mathrm{~g}$ total dietary fibre/kg dry matter

${ }^{2} 12 \mathrm{MJ}$ metabolisable energy, $246 \mathrm{~g}$ total dietary fibre/kg dry matter, added fibre = brewer's grains, lucerne hay, maize cobs, soy hulls or wheat bran

${ }^{3} 11 \mathrm{MJ}$ metabolisable energy, $319 \pm 1.4 \mathrm{~g}$ total dietary fibre/kg dry matter, ingredients screened for maximal contrast in fibre fermentability $=\mathrm{mL}$ fermentation gas $/ \mathrm{kg}$ dry matter

Individual feed insoluble fibre fermentation gas and SCFA production (Table 5) decreased $(P<0.05)$ proportionately in the order of dehulled soybean $\geq$ maize $\geq$ soy hulls $\geq$ maize hominy chop $>$ wheat bran $>$ lucerne hay $\geq$ brewer's grain = maize cob. The botanical influence on dietary insoluble fibre fermentation was reflected in the single standard, single coproduct diets (Table 6). Screening dietary ingredients for HF and LF fibre imposed contrasting fermentability $(P<0.05)$ (Table 7$)$, due largely to differentiated allotment of soy hulls to the HF and brewer's grain to the LF diets. Dietary dilution with coproduct insoluble fibre decreased $(P$ $<0.05)$ fermentability for all except the soy hull and HF fibre. In the individual feed ingredients and in the single product diets, cereal fibre induced proportionately more $(P<0.05)$ propionate and butyrate, and less $(P$ $<0.05)$ acetate, with a greater butyrate shift $(P<0.05)$ for maize fibre. High levels $(P<0.05)$ of proteolytic markers were produced from lucerne (iso-butyrate) and soy hull (iso-valerate) fibre, signifying protein fermentation. Consistent with the differentiated allotment of soy hulls to HF and brewer's grain to LF diets, the HF fibre increased acetate more than the LF fibre, with less butyrate in the HF diet $(P<0.05)$. 
Table 4 Partial multi-enzyme cocktail insoluble fibre digestibility $(n=36)$

\begin{tabular}{|c|c|c|c|c|c|c|c|}
\hline \multirow{2}{*}{ Feedstuffs } & \multirow{2}{*}{ Roxazyme } & \multirow{2}{*}{ Viscozyme } & \multirow{2}{*}{ Roxazyme+Viscozyme } & \multirow{2}{*}{ SEM } & \multicolumn{3}{|c|}{$P$-values } \\
\hline & & & & & Feed & Enzyme & Feed x Enzyme \\
\hline \multicolumn{8}{|l|}{ Ingredients } \\
\hline Brewer's grains & $0.12^{\mathrm{c}}$ & $0.12^{\mathrm{C}}$ & & \multirow{8}{*}{0.010} & \multirow{8}{*}{$<0.001$} & \multirow{8}{*}{$<0.001$} & \multirow{8}{*}{$<0.001$} \\
\hline Maize cobs & $0.05^{\mathrm{ef}}$ & $0.04^{\mathrm{fg}}$ & & & & & \\
\hline Maize hominy chop & $0.02^{g}$ & $0.10^{\mathrm{cd}}$ & & & & & \\
\hline Maize meal & $0.04^{\mathrm{fg}}$ & $0.16^{b}$ & & & & & \\
\hline Dehulled soybean meal & $0.02^{\mathrm{fg}}$ & $0.17^{\mathrm{b}}$ & & & & & \\
\hline Lucerne hay & $0.08^{\mathrm{de}}$ & $0.18^{b}$ & & & & & \\
\hline Soybean hulls & $0.06^{\mathrm{e}}$ & $0.33^{a}$ & & & & & \\
\hline Wheat bran & $0.12^{\mathrm{c}}$ & $0.12^{\mathrm{c}}$ & & & & & \\
\hline \multicolumn{8}{|c|}{ Standard ${ }^{1}$ and single ${ }^{2}$ fibre diets } \\
\hline Standard & $0.03^{d}$ & $0.21^{\mathrm{a}}$ & & \multirow{6}{*}{0.009} & \multirow{6}{*}{$<0.001$} & \multirow{6}{*}{$<0.001$} & \multirow{6}{*}{$<0.001$} \\
\hline Brewer's grains & $0.04^{d}$ & $0.17^{\mathrm{b}}$ & & & & & \\
\hline Maize cobs & $0.03^{d}$ & $0.16^{\mathrm{b}}$ & & & & & \\
\hline Lucerne hay & $0.06^{\mathrm{c}}$ & $0.22^{\mathrm{a}}$ & & & & & \\
\hline Soybean hulls & $0.04^{\mathrm{cd}}$ & $0.21^{\mathrm{a}}$ & & & & & \\
\hline Wheat bran & $0.06^{\mathrm{C}}$ & $0.17^{b}$ & & & & & \\
\hline \multicolumn{8}{|l|}{ Mixed fibre diets ${ }^{3}$} \\
\hline Low fermentability & $0.07^{c}$ & $0.17^{\mathrm{b}}$ & $0.18^{b}$ & \multirow{2}{*}{0.013} & \multirow{2}{*}{$<0.01$} & \multirow{2}{*}{$<0.001$} & \multirow{2}{*}{$<0.001$} \\
\hline High fermentability & $0.04^{\mathrm{C}}$ & $0.20^{\mathrm{b}}$ & $0.25^{\mathrm{a}}$ & & & & \\
\hline
\end{tabular}

${ }^{\text {abcdfg }}$ For each set of means, values that do not share a common superscript are different at $P<0.05$

SEM: Standard error of the mean

${ }_{1}^{1} 13 \mathrm{MJ}$ metabolisable energy, $141 \mathrm{~g}$ total dietary fibre/kg dry matter

$212 \mathrm{MJ}$ metabolisable energy, $246 \mathrm{~g}$ total dietary fibre/kg dry matter, added fibre = brewer's grains, lucerne hay, maize cobs, soy hulls or wheat bran

${ }^{3} 11 \mathrm{MJ}$ metabolisable energy, $319 \pm 1.4 \mathrm{~g}$ total dietary fibre/kg dry matter, ingredients screened for maximal contrast in fibre fermentability $=\mathrm{ml}$ fermentation gas ${ }^{-1} \mathrm{~kg}$ dry matter 
Table 5 Fermentation characteristics $(n=6)$ of individual feed insoluble fibre in pig faecal inoculum

\begin{tabular}{|c|c|c|c|c|c|c|c|c|c|}
\hline \multirow{3}{*}{ Diet } & \multicolumn{7}{|c|}{ Short chain fatty acid production } & \multirow{2}{*}{\multicolumn{2}{|c|}{ Gas production }} \\
\hline & \multicolumn{5}{|c|}{$\%$} & \multirow{2}{*}{$\begin{array}{c}\text { Total }^{1} \\
\text { (mMol/g } \\
\text { dry matter) }\end{array}$} & \multirow{2}{*}{$\begin{array}{c}\text { Ratio } \\
\text { (acetate:propionate:butyrate) }\end{array}$} & & \\
\hline & Acetate & Propionate & Butyrate & $\begin{array}{c}\text { Iso- } \\
\text { butyrate }\end{array}$ & $\begin{array}{c}\text { Iso- } \\
\text { valerate }\end{array}$ & & & $A^{2}$ & $T_{1 / 2}^{3}$ \\
\hline Brewer's grains & $51.4^{d}$ & $39.7^{\mathrm{a}}$ & $7.3^{\mathrm{c}}$ & $0.4^{\mathrm{c}}$ & $1.2^{\mathrm{b}}$ & $2.3^{\mathrm{e}}$ & $52: 40: 08$ & $61.3^{\mathrm{c}}$ & $13.4^{\mathrm{b}}$ \\
\hline Maize cobs & $59.6^{\mathrm{b}}$ & $32.0^{c}$ & $7.9^{\mathrm{c}}$ & $0.2^{c}$ & $0.3^{\mathrm{e}}$ & $2.3^{\mathrm{e}}$ & $60: 32: 08$ & $51.8^{\mathrm{c}}$ & $30.1^{\mathrm{ab}}$ \\
\hline $\begin{array}{l}\text { Maize hominy } \\
\text { chop }\end{array}$ & $51.3^{d}$ & $35.8^{b}$ & $12.1^{b}$ & $0.3^{c}$ & $0.7^{\mathrm{cd}}$ & $4.3^{\mathrm{c}}$ & $52: 36: 12$ & $173.5^{b}$ & $29.8^{\mathrm{ab}}$ \\
\hline Maize meal & $48.8^{\mathrm{e}}$ & $35.4^{\mathrm{b}}$ & $14.8^{\mathrm{a}}$ & $0.3^{\mathrm{c}}$ & $0.7^{\mathrm{cd}}$ & $5.3^{\mathrm{b}}$ & $45: 39: 16$ & $245.2^{\mathrm{ab}}$ & $29.1^{\mathrm{ab}}$ \\
\hline $\begin{array}{l}\text { Dehulled soybean } \\
\text { meal }\end{array}$ & $53.9^{c}$ & $37.4^{\mathrm{ab}}$ & $7.0^{\mathrm{cd}}$ & $0.7^{\mathrm{ab}}$ & $1.0^{\mathrm{bc}}$ & $6.0^{a}$ & 55:38:07 & $299.4^{a}$ & $32.3^{\mathrm{a}}$ \\
\hline Lucerne hay & $61.0^{\mathrm{b}}$ & $32.0^{\mathrm{c}}$ & $5.4^{\mathrm{e}}$ & $0.8^{\mathrm{a}}$ & $0.8^{c d}$ & $2.4^{\mathrm{de}}$ & 62:33:05 & $65.2^{\mathrm{C}}$ & $18.5^{b}$ \\
\hline Soy hulls & $66.7^{\mathrm{a}}$ & $26.1^{d}$ & $5.3^{\mathrm{e}}$ & $0.5^{\mathrm{abc}}$ & $1.5^{\mathrm{a}}$ & $4.5^{c}$ & $72: 23: 05$ & $184.4^{\mathrm{ab}}$ & $20.6^{b}$ \\
\hline Wheat bran & $51.7^{d}$ & $38.8^{\mathrm{a}}$ & $8.5^{c}$ & $0.4^{\mathrm{c}}$ & $0.6^{\mathrm{de}}$ & $2.9^{d}$ & 52:39:09 & $67.6^{\mathrm{c}}$ & $12.7^{b}$ \\
\hline SEM & 1.21 & 0.88 & 0.65 & 0.04 & 0.08 & 0.29 & & 18.11 & 2.95 \\
\hline$P$-values & $<0.0001$ & $<0.0001$ & $<0.0001$ & $<0.0001$ & $<0.0001$ & $<0.0001$ & & $<0.0001$ & $<0.0001$ \\
\hline
\end{tabular}

abcde Means within a column with different superscripts are different at $P<0.05$

SEM: Standard error of the mean

${ }^{1}$ Acetate, propionate, butyrate, iso-butyrate, iso-valerate

${ }^{2}$ A- $\mathrm{mL} / \mathrm{g}$ dry matter at $\mathrm{t}=\infty$

${ }^{3}$ Time (hours), when gas production $=\mathrm{A} / 2$ 
Table 6 Fermentation characteristics $(n=6)$ of standard $^{1}$, and single co-product ${ }^{2}$ dietary insoluble fibre in pig faecal inoculum

\begin{tabular}{|c|c|c|c|c|c|c|c|c|c|}
\hline \multirow{3}{*}{ Diet } & \multicolumn{7}{|c|}{ Short chain fatty acid production } & \multirow{2}{*}{\multicolumn{2}{|c|}{ Gas production }} \\
\hline & \multicolumn{5}{|c|}{$\%$} & \multirow{2}{*}{$\begin{array}{l}\text { Total }^{3} \\
\text { (mMol/g dry } \\
\text { matter) }\end{array}$} & \multirow{2}{*}{$\begin{array}{c}\text { Ratio } \\
\text { (acetate:propionate:butyrate) }\end{array}$} & & \\
\hline & Acetate & Propionate & Butyrate & $\begin{array}{c}\text { Iso- } \\
\text { butyrate }\end{array}$ & $\begin{array}{c}\text { Iso- } \\
\text { valerate }\end{array}$ & & & $A^{4}$ & $T_{1 / 2}^{5}$ \\
\hline${ }^{1}$ Standard & $45.4^{c}$ & $39.7^{\mathrm{a}}$ & $13.5^{\mathrm{a}}$ & $0.4^{\mathrm{b}}$ & 1.5 & 5.0 & $46: 41: 13$ & $205.3^{\mathrm{a}}$ & 25.2 \\
\hline $\begin{array}{l}\text { 'Brewer's } \\
\text { grain }\end{array}$ & $48.6^{\mathrm{b}}$ & $38.3^{\mathrm{ab}}$ & $11.6^{\mathrm{ab}}$ & $0.4^{\mathrm{ab}}$ & 1.1 & 4.3 & 49:39:12 & $126.6^{c}$ & 14.2 \\
\hline${ }^{2}$ Maize cobs & $47.5^{\mathrm{b}}$ & $37.5^{\mathrm{ab}}$ & $12.7^{\mathrm{ab}}$ & $0.5^{\mathrm{ab}}$ & 0.9 & 4.1 & 49:38:13 & $139.8^{\mathrm{c}}$ & 17.8 \\
\hline${ }^{2}$ Lucerne hay & $51.0^{b}$ & $36.1^{\mathrm{bc}}$ & $11.3^{\mathrm{b}}$ & $0.6^{\mathrm{a}}$ & 1.1 & 5.4 & $52: 37: 11$ & $155.2^{\mathrm{bc}}$ & 19.3 \\
\hline${ }^{2}$ Soybean hulls & $53.0^{\mathrm{a}}$ & $34.8^{\mathrm{c}}$ & $10.7^{\mathrm{b}}$ & $0.4^{\mathrm{ab}}$ & 1.1 & 5.2 & $54: 35: 11$ & $187.6^{\mathrm{ab}}$ & 23.4 \\
\hline${ }^{2}$ Wheat bran & $50.0^{b}$ & $37.8^{\mathrm{b}}$ & $10.9^{\mathrm{ab}}$ & $0.5^{\mathrm{ab}}$ & 0.9 & 4.4 & $51: 38: 11$ & $141.0^{c}$ & 21.8 \\
\hline SEM & 0.63 & 0.41 & 0.28 & 0.03 & 0.09 & 0.19 & & 6.36 & 1.03 \\
\hline$P$-values & $<0.0001$ & $<0.0001$ & $<0.01$ & 0.22 & 0.35 & $<0.05$ & & $<0.001$ & $<0.05$ \\
\hline
\end{tabular}

${ }^{\mathrm{abc}}$ For each substrate, means within a column with different superscripts are different at $P<0.05$

SEM: Standard error of the mean

${ }_{1}^{1} 13 \mathrm{MJ}$ metabolisable energy, $141 \mathrm{~g}$ total dietary fibre/kg dry matter maize-soybean diet

$212 \mathrm{MJ}$ metabolisable energy, $246 \mathrm{~g}$ total dietary fibre/kg dry matter added fibre = brewer's grains, lucerne hay, maize cobs, soy hulls or wheat bran

${ }^{3}$ Acetate, propionate, butyrate, iso-butyrate, iso-valerate

${ }^{4}$ Gas production ( $\mathrm{mL} / \mathrm{g}$ dry matter) at $\mathrm{t}=\infty$

${ }^{5}$ Time (hours), when gas production $=\mathrm{A} / 2$ 
Table 7 Fermentation characteristics $(n=8)$ of standard ${ }^{1}$, and high versus low fermentability insoluble dietary fibre in pig faecal inoculum

\begin{tabular}{|c|c|c|c|c|c|c|c|c|c|}
\hline \multirow{3}{*}{ Diet } & \multicolumn{7}{|c|}{ Short chain fatty acid production } & \multirow{2}{*}{\multicolumn{2}{|c|}{ Gas production }} \\
\hline & \multicolumn{5}{|c|}{$\%$} & \multirow{2}{*}{$\begin{array}{l}\text { Total }^{3} \\
\text { (mMol/g dry } \\
\text { matter) }\end{array}$} & \multirow{2}{*}{$\begin{array}{c}\text { Ratio } \\
\text { (Acetate:propionate:butyrate) }\end{array}$} & & \\
\hline & Acetate & Propionate & Butyrate & $\begin{array}{c}\text { Iso- } \\
\text { butyrate }\end{array}$ & $\begin{array}{c}\text { Iso- } \\
\text { valerate }\end{array}$ & & & $A^{4}$ & $T_{1 / 2}^{5}$ \\
\hline $\begin{array}{l}{ }^{2} \text { High } \\
\text { fermentability }\end{array}$ & $55.2^{\mathrm{a}}$ & $33.8^{\mathrm{b}}$ & $9.5^{\mathrm{b}}$ & 0.5 & 1.1 & $5.0^{\mathrm{a}}$ & 51:38:11 & $159.5^{\mathrm{a}}$ & 27.4 \\
\hline $\begin{array}{l}\text { 'Low } \\
\text { fermentability }\end{array}$ & $50.5^{\mathrm{b}}$ & $37.2^{\mathrm{ab}}$ & $10.9^{\mathrm{ab}}$ & 0.5 & 1.0 & 3.6 & $48: 39: 13$ & $96.6^{\mathrm{b}}$ & 29.8 \\
\hline
\end{tabular}

${ }^{\mathrm{ab}}$ For each set of means, values within a column with different superscripts are different at $P<0.05$

SEM: Standard error of the mean

${ }^{1} 13 \mathrm{MJ}$ metabolisable energy, $141 \mathrm{~g}$ total dietary fibre/kg dry matter

${ }^{2} 11 \mathrm{MJ}$ metabolisable energy, $319 \pm 1.4 \mathrm{~g}$ total dietary fibre/ $\mathrm{kg}$ dry matter, feed ingredients screened for maximal contrast in fibre fermentability (mL fermentation gas ${ }^{-1} \mathrm{~kg}$ dry matter)

${ }^{3}$ Acetate, propionate, butyrate, iso-butyrate, iso-valerate

${ }^{3}$ Gas production $(\mathrm{mL} / \mathrm{g}$ dry matter) at $\mathrm{t}=\infty$

${ }^{4}$ Time (hours), when gas production $=\mathrm{A} / 2$ 


\section{Discussion}

The study examined how botanical factors and fermentation-based dilution of co-product feeds into high fibre maize-soybean growing-pig diets affect exogenous enzymatic and fermentative insoluble dietary fibre degradation, with the goal to improve the nutritional efficacy of such complex diets. Differentiated occurrence of NSP in plant tissues among feed-grade grain-processing co-products is well documented (Bach Knudsen, 2014). Cellulose is ubiquitous with chemical homogeneity, but high quantitative variance across plant genera (Bach Knudsen, 2014). Fibre in the gramineae is dominated by arabinoxylans (rye, wheat, maize and sorghum) and $\beta$-glucans (oats and barley) (Bach Knudsen, 2014). Legume fibre is similarly highly heterogenic, consisting mostly of pectins, xyloglucans and mannans (Jha \& Leterme, 2012; Bach Knudsen, 2014; Aumiller et al., 2015). Plant iNSP are structurally differentiated moieties at the primary, secondary and tertiary domains of the cell wall architecture (e.g. reviews by McDougall et al., 1996, Collins et al., 2010, Bach Knudsen, 1997, 2014, Pedersen et al., 2015; Bach Knudsen et al., 2016). In the study test feed range, chemical variation in NSP was generically indicated by different neutral and acid detergent fibre, hemi-cellulose, ADL, cellulose, soluble versus insoluble fractions, with the quantitative fibre occurrence reflected in the total dietary fibre (Tables $1 \& 2 b$ ). The variable fibre content was implicated indirectly in the different enzymatic (pepsin-pancreatin) indigestibility (Table 3).

In line with previous studies (Willamil et al., 2012; Jha et al., 2015; Kong et al., 2015; Kwon et al., 2015; Bach Knudsen, 2015), in the present study, there were strong influences of the fibre source on both enzymatic and fermentative degradation of dietary fibre. Overall, enzymatic insoluble fibre degradation was highly variable, and limited (0.02 - 0.33). Enzyme-co-product interaction on fibre digestibility in feed ingredients, which was expressed in complete single co-product diets, confirmed expected differentiated enzyme-substrate specificity. Roxazyme expressed notably low affinity for the basal maize and soybean fibre types, which suggested limited efficacy on such diets owing to lack of substrate, which was in line with previous studies (e.g. Willamil et al., 2012; Pedersen et al., 2015; Park et al., 2016b). Though enzyme fibrolytic efficacy seems generally higher on temperate cereal fibre, for which most exogenous enzymes were developed, inconsistent pig responses are attributed partly to undefined interactions of animal and dietary factors in various experiments (Swiatkiewicz et al., 2016). Of an array of commercial fibrolytic enzyme cocktails, none improved the utilization of co-product-enriched maize-based diets (Kerr \& Shurson, 2013). In contrast to the glycoside 10 xylanases, the glycoside 11 family of xylanases expressed greater specificity (Biely et al., 1997; Paes et al., 2012), which probably accounted for the low efficacy of xylanases in Roxazyme in cleaving the highly substituted maize arabinoxylans. Maize arabinoxylans exhibit greater frequency of random arabinose, D-glucuronic acid and acetyl group substitution into the $\beta$-linked D-xylose chains with feruloylated arabinose residues forming cross-links to create heterogeneous recalcitrant complexes (Pedersen et al., 2014). The analytical markers for such arabinoxylans are high arabinose/xylose and uronic acid/xylose ratios (Pedersen et al., 2014). The bulk of any in vitro enzymatic digestibility of maize fibre could be starch, and not the hull iNSP (Park et al., 2016a). Unlike the extremely diverse pig gut microbial enzyme activities, only limited activities are expressed in manufactured enzyme cocktails. Therefore, greater diversity of gut microbial enzyme activities theoretically affords better digestion owing to synergistic/complementary degradation (Ghimire et al., 2016), which was demonstrated by the combination of the carbohydrase enzyme cocktails in degradation of HF, but not of LF. It is against this background that the efficacy of current enzymes, particularly for the iNSP-rich maize diet, remains equivocal (Zijlstra et al., 2010; Bedford \& Cowieson, 2012; Willamil et al., 2012, Rho et al., 2018). Futurist enzymes could be tailored (Bindelle et al., 2011; Bedford \& Cowieson, 2012) to diet specific or broad potency.

Fermentation gas and total SCFA jointly predict fermentability (Jonathan et al., 2012). In the present study, they similarly differentiated feed fibre fermentability, and both detected significantly induced controlHF-LF fermentability contrasts. However, the prediction was dissimilar for the single fibre diets, which had similar SCFA, but different gas yields. Jonathan et al. (2012) reported significant correlation of the gas and SCFA in the early stages of fermentation, and not by the end of fermentation. The anomaly was attributed to fermentation pathways that differently partition carbon into SCFA versus gases. Gas formation is not universal to anaerobic bacteria, least gas producing of which are the probiotic Bifidobacterium and Lactobacilli spp (Rowland et al., 2018). For example, lactate, which is metabolized early during fermentation to yield propionate, butyrate and gases was detected after 72 hours of fermentation of oat $\beta$-glucans and inulin, and not in other substrates. Further, the $\mathrm{CO}_{2}$ produced from bicarbonate buffering is subject to both quantitative and qualitative SCFA production (Bindelle et al., 2011). Ideally, gas and SCFA prediction of fermentability should be qualitatively supported by measurement of intermediate and endpoint metabolites, and the residual substrate (Jonathan et al., 2012). Continuous fermentation models (Tanner et al., 2014) might provide better simulation of the dynamics of porcine colon fermentation. In the present study, 
considering both measures of fermentability, similar to the enzymatic digestion, the fibre type strongly influenced its fermentation characteristics, which was consisted with previous studies (Jha et al., 2011; Jha \& Leterme, 2012; Jonathan et al., 2012; Jha \& Berrocoso, 2015). The basal (maize grain, dehulled soybean) dietary fibre were the most fermentable, which ensured that, despite co-product addition, all test diets, which were maize-soybean diets remained highly fermentable. In maize grain, endosperm arabinoxylans are chemically simplest (Pedersen et al., 2015) and therefore highly fermentable (Bach Knudsen, 2014). Soy iNSP are highly fermentable (McDougall et al., 1996) owing to extensive branching, which provides abundant cleaving sites for microbial enzymes (Jonathan et al., 2012). The least fermented co-products are those in which the secondary plant tissue is dominated by cellulose and the most complex non-cellulosic iNSP (Bach Knudsen et al., 2016), and the most lignified (Tables 1 and 2).

The butyrate-type fermentation of cereal fibre is attributed to its arabinoxylans (Chen et al., 2014; Ivarsson et al., 2014). The acetate-type fermentation of legume fibre is linked to polyuronides (Jonathan et al., 2012; Chen et al., 2014; Ivarsson et al., 2014). Intense fermentation with substantial BUT shift for maize and its hominy chop could be attributed to a concentration of resistant starch (Jonathan et al., 2012; Giuberti et al., 2013; He et al., 2017). Resistant starch is fully recovered in the filtered pepsin-pancreatin residues (Giuberti et al., 2013). Its fermentation is beneficially bifidogenic (Fouhse et al., 2015). In the distal colon, an energy-protein imbalance may occur owing to depletion of fermentable NSP or excessive undigested exogenous and endogenous protein, which forces bacteria into amino acid fermentation, with deleterious effects on the intestinal barrier function (Pieper et al., 2016). Colon microbiota exhibit preference for few of the natural amino acids, including the branched-chain amino acids (Dai et al., 2011), which uniquely produce BCFA. BCFA also modulate epithelial cell metabolism and the mucosal immune system (Blachier et al., 2007; Neis et al., 2015). In this study, proteolytic colon fermentation was evident in the legume co-products, which yielded more I-BUT (lucerne) or iso-valerate (soy hulls). Proteolytic fermentation probably occurred in the terminal stages of fermentation, consistent with distal colonic fermentation (Rowland et al., 2018), after depletion of the fermentable iNSP. In addition to toxic metabolites (Cone et al., 2005; De Lange et al., 2010; Jha \& Leterme, 2012), proteolytic fermentation favours pathogenic microbiota (Pieper et al., 2016; Yao et al., 2016). In vivo, the dietary protein level, viscosity, particle size, bulking, anti-nutritional compounds, upper gut passage rate, feed intake and stress are among key factors that are associated with dietary protein wastage, and subsequent deleterious colon protein fermentation (Pieper et al., 2016). In fibre extracts from the complete diets, concentration of maize resistant starch probably excluded amino acid fermentation (He et al., 2017).

In vitro co-product screening is necessarily subject to biologic and cost-benefit validation in responsebased experimentation. The control diet of the present study was the typical maize-soybean growing pig diet in which these basal ingredients were the only and therefore exclusive sources of dietary energy. Apart from lucerne hay, these co-products were largely of insignificant protein value, such that the primary benefit of their dilution into the standard diet was to substitute fermentable iNSP for expensive starch and fat. Starch and fat were theoretically most displaced by targeting feed fermentable fibre, which was demonstrated in the HF/LF diets. Given the high fibre, dietary energy should ideally be quantified in net energy terms to accommodate fibre-specific energy wastage (Noblet \& Le Goff., 2001; De Lange et al., 2010; Gao et al., 2015; Velayudhan et al., 2015). Similarly, a truly digestible essential amino acid approach accounts for fibrespecific endogenous amino acid wastage (Zhu et al., 2005; Libao-Mercado et al., 2006; 2007), and for the trade-off between fermentative loss (Columbus et al. 2010), and microbial synthesized digestible amino acids (Torrallardona et al., 2003; Zhu et al., 2005; 2007; Libao-Mercado et al., 2009). The HF-LF approach to diet formulation could theoretically incorporate iNSP, whose properties induce beneficial probiotic bacteria and SCFA (Giuberti et al., 2013). Considering the strong influence of co-product botany on the dietary SCFA pattern, the objective to screen the ingredients to influence SCFA ratios may therefore be practical. However, in vivo, the stoichiometry of SCFA production is more complex. While generically related to the feed NSP composition (Awati et al., 2005), the SCFA pattern is further specifically controlled by the constitutive neutral sugars (Ivarsson et al., 2014). Fermentation stoichiometry should depend on the net fermentative redox gradient toward maximal microbial energy extraction (Macfarlane \& Macfarlane, 1993; 2003), though gut fermentation pathways are complexly fluid, subject to substrate availability, in relation to interspecies competition and cross feeding (Macfarlane \& Macfarlane, 2003). In vivo, additional complicating variables include endogenous secretions into the gut, and differential SCFA clearance by absorption (Den Besten et al., 2013).

In principle, the sensitivity of both fibre enzymatic hydrolysis and fermentation assays to botanical factors supported the application of the in vitro model to strategic co-product feeding. Subject to testing across a wider range of substrates for empirical clarity to the underpinning monomeric NSP chemistryfermentation relationships, the study presents conceptual and procedural frameworks for targeting maximal fermentable energy, physiologically beneficial fermentation, and for matching enzymes to dietary NSP, 
integral to the least-cost strategic formulation of co-product, iNSP-rich diets. Co-product and enzyme screening should be guided by prevailing dietary, animal and economic factors.

\section{Conclusion}

High-level fermentation-based dietary inclusion of co-product feeds in maize-soybean growing pig diets altered fibrolytic enzymatic efficacy, fermentability and the SCFA pattern according to the botanical origin, with important biogenic implications. Roxazyme expressed low potency in degrading maize and soybean insoluble fibre, which casts doubt on its potency to improve the nutritional efficacy in such diets. The in vitro approach could be a practical tool to match enzyme products to specific diets, and to screen co-products for calibrated dietary incorporation to promote maximal fermentative fibre digestion, and induce beneficial fermentation in low-cost, fibrous maize-soybean-co-product diets.

\section{Acknowledgements}

The authors express gratitude to the South African Pork Producers' Organisation (SAPPO) and to the National Research Foundation of South Africa, which funded the project jointly through the Technology and Human Resources for Industry Programme (THRIP) Project (Grant number TP2010072700024). Acknowledgement is also extended to the Animal Production Institute of the Agricultural Research Council of South Africa, and to Tshwane University of Technology, for providing the equipment and facilities for the experiments.

\section{Authors' Contributions}

The research reported was jointly designed, conducted, and interpreted by all the cited authors, who have read and approved the manuscript.

\section{Conflict of Interest Declaration}

None of the authors declare any competing interests.

\section{References}

Agyekum, A.K., Kiarie, E., Walsh, M.C. \& Nyachoti, C.M., 2016. Postprandial portal fluxes of essential amino acids, volatile fatty acids, and urea-nitrogen in growing pigs fed a high-fibre diet supplemented with a multi-enzyme cocktail. J. Anim. Sci. 94, 3771-3785.

Agyekum, A.K. \& Nyachoti, C.M., 2017. Nutritional and metabolic consequences of feeding high fibre diets to Swine - A review. Anim. Feed Sci. Technol. 234, 88-100.

Anguita, M., Canibe, N., Perez, J.F. \& Jensen, B.B., 2006. Influence of the amount of dietary fibre on the available energy from hindgut fermentation in growing pigs: Use of cannulated pigs and in vitro fermentation. J. Anim. Sci. 84, 2766-2778.

AOAC, 2006. Official methods of analysis. Association of Official Analytical Chemists International, Washington, DC, USA.

Aumiller, T., Mosenthin, R. \& Weiss, E., 2015. Potential of cereal grains and grain legumes in modulating pigs' intestinal microbiota - A review. Livest. Sci. 17, 16-32.

Awati, A., Konstantinov, S.R., Williams B.A., Akkermans A.D.L., Bosch, M.W., Smidt, H. \& Verstegen, M.W.A., 2005. Effect of substrate adaptation on the microbial fermentation and microbial composition of faecal microbiota of weaning piglets studied in vitro. J. Sci. Food Agr. 85, 1765-1772.

Bach Knudsen, K.E., 1997. Carbohydrate and lignin contents of plant materials used in animal feeding. Anim. Feed Sci. Technol. 67, 319-338.

Bach Knudsen, K.E., 2014. Fibre and non-starch polysaccharide content and variation in common crops used in broiler diets. Poult. Sci. 93, 2380-2393.

Bach Knudsen, K.E., 2015. Microbial degradation of whole-grain complex carbohydrates and impact on short-chain fatty acids and health. Adv. Nutr. 6, 206-213.

Bach Knudsen, K.E., Lærke, H.N., Ingerslev, A.K., Hedemann, M.S., Nielsen, T.S. \& Theil, P.K., 2016. Carbohydrates in pig nutrition - Recent advances. J. Anim. Sci. 94, 1-11.

Bedford, M.R., 2000. Exogenous enzymes in monogastric nutrition - their current value and future benefits. Anim. Feed Sci. Technol. 86, 1-13.

Bedford, M.R. \& Cowieson, A.J., 2012. Exogenous enzymes and their effects on intestinal microbiology. Anim. Feed Sci. Technol. 173, 76-85.

Biely, P., Vr`sanská, M., Tenkanen, M. \& Kluepfel, D., 1997. Endo- $\beta-1$, 4-xylanase families: differences in catalytic properties. J. Biotechnol. 57, 151-166.

Bindelle, J., Buldgen, A., Boudry, C. \& Leterme, P., 2007. Effect of inoculum and pepsin-pancreatin hydrolysis on fibre fermentation measured by the gas production technique in pigs. Anim. Feed Sci. Technol. 132, 111-122.

Bindelle, J., Carlos, R.P., Montoya, A., Van Kessel, A.G. \& Leterme, P., 2011. Non-starch polysaccharide-degrading enzymes alter the microbial community and the fermentation patterns of barley cultivars and wheat products in an in vitro model of the porcine gastrointestinal tract. FEMS Microb. Ecol. 76, 553-563. 
Blachier, F., Mariotti, F., Huneau. J.F. \& Tomé D., 2007. Effects of amino acid-derived luminal metabolites on the colonic epithelium and physiopathological consequences. Amino Acids 33, 547-562.

Boisen, S. \& Fernandez, J.A., 1997. Prediction of the total tract digestibility of energy in feedstuffs and pig diets by in vitro analyses. Anim. Feed Sci. Technol. 68, 277-286.

Byrne, C.S., Chambers, E.S. Morrison, D.J. \& Frost, G., 2015. The role of short chain fatty acids in appetite regulation and energy homeostasis. Int. J. Obesity 39, 1331-1338.

Celi, P., Viviane, V., Estefania, P.C. Jerome, S. \& Kluenter, A-M., 2018. Biomarkers of gastrointestinal functionality in animal nutrition and health. Anim. Feed Sci. Technol. https://doi.org/10.1016/j.anifeedsci.2018.07.012.

Chen, H., Mao, X.B., Che, L.Q., Yu, B., He, J., Yu, J., Han, G.Q., Huang, Z.Q. Zheng, P. \& Chen, D.W., 2014. Impact of fibre types on gut microbiota, gut environment and gut function in fattening pigs. Anim. Feed Sci. Technol. 195, 101-111.

Choct, M., 2006. Enzymes for the feed industry: past, present and future. Wrld. Poult. Sci. J. 62, 5-16.

Collins, H.M., Burton, R.A., Topping, D.L., Liap, M., Bacic, A. \& Fincher, D., 2010. Variability in fine structures of noncellulosic polysaccharides from cereal grains; Potential importance in human health and nutrition. Cereal Chem. 87 (4), 272-282.

Columbus, D., Cant, J.P. \& De Lange, C.F.M., 2010. Estimating fermentative amino acid losses in the upper gut of pigs. Livest. Sci., 133, 124-127.

Cone, J.W., Jongbloed, A.W., Gelder, A.H.V. \& Lange, L.D., 2005. Estimation of protein fermentation in the large intestine of pigs using a gas production technique. Anim. Feed Sci. Technol. 123-124, 463-472.

Dai, Z., Wu, G. \& Zhu, W., 2011. Amino acid metabolism in intestinal bacteria: links between gut ecology and gut health. Front. Biosci. 16, 1768-1786.

Daly, K. \& Shirazi-Beechey, S.P., 2006. Microarray analysis of butyrate regulated genes in colonic epithelial cells. DNA Cell Biol. 25, 49-62.

De Vries, S., Pustjens, A.M., Schols, H.A., Hendriks, W.H. \& Gerrits, W.J.J., 2012. Improving digestive utilization of fibre-rich feedstuffs in pigs and poultry by processing and enzyme technologies: A review. Anim. Feed Sci. Technol. 178, 123-138.

De Lange, C.F., Pluske, J., Gong, J. \& Nyachoti C.M., 2010. Strategic use of feed ingredients and feed additives to stimulate gut health and development in young pigs. Livest. Sci. 134, 124-134.

Den Besten, G., Van Eunen G, Groen, A.K, Venema, K., Reijngoud, D. \& Bakker, B.M., 2013. The role of short-chain fatty acids in the interplay between diet, gut microbiota, and host energy metabolism. J. Lipid Res. 54, 235-2340.

Fouhse, J.M., Ganzle, M.G., Regmi, P.R., Van Kempen, T.A.T. \& Zijlstra, R.T., 2015. High amylose starch with low in vitro digestibility stimulates hindgut fermentation and has a bifidogenic effect in weaned pig. J. Nutr. 145, 2464-2470.

France, J., Dhanoa, M.S., Theodorou, M.K., Lister, S.J., Davies, D.R. \& Isac, D., 1993. A model to interpret gas accumulation profiles associated with in vitro degradation of ruminant feeds. Biol. 163, 99-111.

Fushimi, T., Suruga, K., Oshima, Y., Fukiharu, M., Tsukamoto, Y., Goda, T., 2006. Dietary acetic acid reduces serum cholesterol and triacylglycerols in rats fed a cholesterol-rich diet. Br. J. Nutr. 95, 916-924.

Gao, L., Chen, L., Huang, Q., Meng, L., Zhong, R., Liu, C., Tang, C. \& Zhang, H., 2015. Effect of dietary fibre type on intestinal nutrient digestibility and hindgut fermentation of diets fed to finishing pigs. Livest. Sci. 174, 53-58.

Ghimire, P.S., Ouyang, H., Wang, Q, Luo, Y. Shi, B, Yang, J., Lü, Y. \& Jin, C., 2016. Insight into enzymatic degradation of corn, wheat, and soybean cell wall cellulose using quantitative secretome analysis of Aspergillus fumigatus. J. Proteome Res. 15 (12), 4387-4402.

Giuberti, G., Gallo A., Moschini, M. \& Masoero, F., 2013. In vitro production of short-chain fatty acids from resistant starch by pig faecal inoculum. Animal 7, 1446-1453.

Groot, J.C.J., Cone, J.W., Williams, B.A., Debersaques, F.M.A. \& Lantinga, E.A., 1996. Multiphasic analysis of gas production kinetics for in vitro fermentation of ruminant feeds. Anim. Feed Sci. Technol. 64, 77-89.

Gutierrez, N.A., Kerr, B.J. \& Patience, J.F., 2013. Effect of insoluble-low fermentable fiber from corn ethanol distillation origin on energy, fiber, and amino acid digestibility, hindgut degradability of fiber, and growth performance of pigs. J. Anim. Sci. 91, 5314-5325.

Hamer, H.M., Jonkers D., Venema, K., Vanhoutvin, S., Troost, F.J. \& Brummer, R.J., 2008. Review article: the role of butyrate on colonic function. Aliment. Pharmacol. Ther. 27, 104-119.

He, X., Sun, W., Ge, T., Mu, C. \& Zhu, W., 2017. An increase in corn resistant starch decreases protein fermentation and modulates gut microbiota during in vitro cultivation of pig large intestinal inocula. Anim. Nutr. 3, 219-224

Ivarsson, V., Roos, S., Liu, H.Y. \& Lindberg, J.E., 2014. Fermentable non-starch polysaccharides increase the abundance of Bacteroides-Prevotella-Porphyromonas in ileal microbial community of growing pigs. Animal 8, 1777-1787.

Iyayi, E.A. \& Adeola, O., 2015. Quantification of short-chain fatty acids and energy production from hindgut fermentation in cannulated pigs fed graded levels of wheat bran. J. Anim. Sci. 10, 4781-4782.

Jha, R. \& Berrocoso, J.D., 2015. Dietary fibre utilization and its effects on physiological functions and gut health of swine: A review. Animal 9, 1441-1452.

Jha, R. \& Berrocoso, J.D., 2016. Dietary fibre and protein fermentation in the intestine of swine and their interactive effects on gut health and on the environment: A review. Anim. Feed Sci. Technol. 212, 18-26.

Jha, R. \& Leterme, P., 2012. Feed ingredients differing in fermentable fibre and indigestible protein content affect fermentation metabolites and faecal nitrogen excretion in growing pigs. Animal 6, 603-611. 
Jha, R., Bindelle, J., Van Kessel, A. \& Leterme, P., 2011. In vitro fibre fermentation of feed ingredients with varying fermentable carbohydrate and protein levels and protein synthesis by colonic bacteria isolated from pigs. Anim. Feed Sci. Technol. 165, 191-200.

Jha, R., Woyengo, T.A., Li, J., Bedford, M.R., Vasanthan, T. \& Zijlstra, R.T., 2015. Enzymes enhance degradation of the fibre-starch-protein matrix of distillers dried grains with solubles as revealed by a porcine in vitro fermentation model and microscopy. J. Anim. Sci. 93, 1039-1951.

Jonathan, M.C., Van den Borne, J.G.C., Van Wiechen, P., Da Silva, C.S., Schols, H.A. \& Gruppen, H., 2012. In vitro fermentation of 12 dietary fibres by faecal inoculum from pigs and humans. Food Chem. 133, 889-897.

Kerr, B.J. \& Shurson, G.C., 2013. Strategies to improve fibre utilization in swine. J. Anim. Sci. Biotechnol. 4, 11-23.

Kong, C, Park, C.S \& Kim, B.G., 2015. Effects of an enzyme complex on in vitro dry matter digestibility of feed ingredients for pigs. SpringerPlus 4, 261.

Kwon, W.B., Park, S.K, Kong, C. \& Kim, B.G., 2015. The effect of various inclusion levels of $\beta$-mannanase on nutrient digestibility in diets consisting of corn, soybean meal and palm kernel expellers fed to growing pigs. Am. J. Anim. Vet. Sci. 10, 9-13.

Lee, J.W., Patterson, R. \& Woyengo, T.A., 2018. Porcine in vitro degradation and fermentation characteristics of canola co-products without or with fiber-degrading enzymes. Anim. Feed Sci. Technol. 241, 133-140

Libao-Mercado, A.J., Yin, Y., Van Eys, J. \& de Lange, C.F.M., 2006. True ileal amino acid digestibility and endogenous ileal amino acid losses in growing pigs fed shorts of casein-based diets. J. Anim. Sci. 84, 1351-1361.

Libao-Mercado, A.J., Zhu, C.L., Fuller, M.F., Rademacher, M., Seve, B. \& De Lange, C.F.M., 2007. Effect of feeding fermentable fibre on synthesis of total and mucosal protein in the intestine of the growing pig. Livest. Sci. 109, 125-128.

Libao-Mercado, A.J., Zhu, C.L., Cant, J.P., Lapierre, H., Thibault, J., Sève, B., Fuller, M.F. \& De Lange, C.F.M., 2009. Dietary and endogenous amino acids are the main nitrogen sources for microbial protein synthesis in the upper gut of pigs. J. Nutr. 139, 1088-1094.

Macfarlane, G.T. \& Macfarlane, S., 1993. Factors affecting fermentation reactions in the large bowel. Proc. Nutr. Soc. 52, 367-373.

Macfarlane, S. \& Macfarlane, G.T., 2003. Regulation of short-chain fatty acid production. Proc. Nutr. Soc. 62, 67-72.

Mangian, H.F. \& Tappenden, K.A., 2009. Butyrate increases GLUT2 mRNA abundance by initiating transcription in Caco2-BBe cells. J. Parenter. Enteral. Nutr. 33, 607-617.

Marten, G.C. \& Barnes, R.F., 1980. Prediction of energy digestibility of forages with in vitro rumen fermentation and fungal enzyme systems, in standardization of analytical methodology for feeds. Proceedings of a workshop in Ottawa, Canada. 12-14 March 1979. IDRC, Ottawa, Ontario.

McDougall, G.J., Morrison, I.M., Stewart, D. \& Hillman, J.R., 1996. Plant cell walls as dietary fibre: Range, structure, processing and function. J. Sci. Food Agr. 70, 133-150.

Molist, F., Gómez de Segura, A., Pérez, J.F., Bhandari, S.K., Krause, D.O. \& Nyachoti, C.M., 2010. Effect of wheat bran on the health and performance of weaned pigs challenged with Escherichia coli K88+. Livest. Sci. 133, 214-217.

Nahm, K.H., 2003. Influences of Fermentable carbohydrates on shifting nitrogen excretion and reducing ammonia emission of pigs. Crit. Rev. Env. Sci. Technol. 33 (2), 165-186.

National Research Council, 1998. Nutrient Requirements for Swine. 10th edition. National Academy Press, Washington DC., USA.

Neis, E, P.J.G., Dejong, C.H.C. \& Rensen, S.S., 2015. The role of microbial amino acid metabolism in host-metabolism. Nutrients 7, 2930-2946.

Noblet, J. \& Le Goff, G., 2001. Effect of dietary fibre on the energy value of feeds for pigs. Anim. Feed Sci. Technol. 90, $35-52$.

Noblet, J. \& Perez, J.M., 1993. Prediction of digestibility of nutrients and energy values of pig diets from chemical analysis. J. Anim. Sci. 71, 3389-3398.

Paes, G., Berrin, J.G. \& Beaugrand, J., 2012. GH11 xylanases: structure/function/properties relationships and applications. Biotechnol. Adv. 30, 564-592.

Park, K.R., Park, C.S. \& Kim B.G., 2016a. An enzyme complex increases in vitro dry matter digestibility of corn and wheat in pigs. SpringerPlus 5, 598. Https://doi.org/10.1186/s40064-016-2194-5

Park, C.S., Park, I. \& Kim, B.G., 2016b. Effects of an enzyme cocktail on digestible and metabolizable energy concentrations in barley, corn, and wheat fed to growing pigs. Livest. Sci. 187, 1-5.

Pedersen, M.B., Dalsgaard, S., Bach Knudsen, K.E., Yua, S. \& Lærke, H.N., 2014. Compositional profile and variation of distillers dried grains with solubles from various origins with focus on non-starch polysaccharides. Anim. Feed Sci. Technol. 197, 130-141.

Pedersen, M.B., Dalsgaard, S., Arenta, S., Lorentsena, R., Bach Knudsen, K.E.B., Yua, S. \& Lærke, H.N., 2015. Xylanase and protease increase solubilization of non-starch polysaccharides and nutrient release of corn- and wheat distillers dried grains with solubles. Biochem. Eng. J. 98, 99-106.

Pieper, R.C., Villodre Tudela, C.V., Taciak, M., Bindelle, J.F., Pérez, J.F. \& Zentek, J., 2016. Health relevance of intestinal protein fermentation in young pigs. Anim. Health. Res. Rev. 17 (2), 137-147.

Rho, Y., Jarie, E. \& de Lange, C.F.M., 2018. Nutritive value of corn distiller's dried grains with solubles steeped without or with exogenous feed enzymes for $24 \mathrm{~h}$ and fed to growing pigs. J. Anim. Sci. 96, 2352-2360. 
Rideout, T.C., Fan, M.Z., Cant, J.P., Wagner-Riddle, C. \& Stonehouse, P., 2004. Excretion of major odour causing and acidifying compounds in response to dietary fibre supplementation of chicory in growing pigs. J. Anim. Sci. 82, 1678-1684.

Ríos-Covián, D., Ruas-Madiedo, P., Margolles, A., Gueimonde, M., de los Reyes-Gavilán, C.G. \& Salazar, N., 2016. Intestinal short chain fatty acids and their link with diet and human health. Front. Microbiol. 7, 185. Doi: 10.3389/fmicb.2016.00185

Rowland, I., Gibsonm, G., Heinken, A., Scott, K., Swann, J., Thiele, I. \& Tuohy, K., 2018. Gut microbiota functions: metabolism of nutrients and other food components. Eur. J. Nutr. 57, 1-24.

Simpson, H.L. \& Campbell, B.J., 2015. Review article: Dietary fibre-microbiota interactions. Aliment. Pharmacol. Ther. 42, 158-179.

Sleeth, M.L., Thompson, E.L., Ford, H.E., Zac-Varghese, S.E.K. \& Frost, G., 2010. Free fatty acid receptor 2 and nutrient sensing a proposed role for fibre, fermentable carbohydrates and short-chain fatty acids in appetite regulation. Nutr. Res. Rev. 23, 135-145.

Statistical Analysis Systems Institute 2010. Statistical analysis systems. Version 9.3. SAS Institute Inc., Cary, NC.

Swiatkiewicz, S., Swiatkiewicz, M., Arczewska-Wlosek, A. \& Jozefiak, D., 2016. Efficacy of feed enzymes in pig and poultry diets containing distillers dried grains with solubles: A review. J. Anim. Physiol. Anim. Nutr. 100, 15-26.

Tanner SA, Zihler Berner, A, Rigozzi, E, Grattepanche, F., Chassard. C, et al. 2014. In vitro Continuous Fermentation Model (PolyFermS) of the swine proximal colon for simultaneous testing on the same gut microbiota. PLOS ONE 9(4): e94123. https://doi.org/10.1371/journal.pone.0094123

Theil, P.K., Jørgensen, H., Serena, A., Hendrickson, J. \& Bach Knudsen, K.E., 2011. Products deriving from microbial fermentation are linked to insulinaemic response in pigs fed breads prepared from whole-wheat grain and wheat and rye ingredients. Br. J. Nutr. 105, 373-383.

Tonel, I., Pinho, M., Lordelo, M.M., Cunha, L.F., Garres, P. \& Freire, J.P.B., 2010. Effect of butyrate on gut development and intestinal mucosa morphology of piglets. Livest. Sci. 133, 222-224.

Torrallardona, D., Harris, C.I. \& Fuller, M.F., 2003. Pigs' gastrointestinal microflora provides them with essential amino acids. J. Nutr. 133, 1127-1131.

Van Soest, P.J., Robertson, J.B. \& Lewis, B.D., 1991. Methods of dietary fibre neutral detergent fibre and non-starch polysaccharides in relation to animal nutrition. J. Dairy Sci. 74, 3583-3597.

Velayudhan, D.E., Kim, I.H. \& Nyachoti, C.M., 2015. Characterization of dietary energy in swine feed and feed ingredients: A review of recent research results. Asian-Australas. J. Anim. Sci. 28, 1-13.

Verbeke, K.A., Boobis, A.R., Chiodini, A., Edwards, C.A., Franck, A., Kleerebezem, M., Nauta, A., Raes, J., Van Tol., E.A.F. \& Tuohy, K.M., 2015. Towards microbial fermentation metabolites as markers for health benefits of prebiotics. Nutr. Res. Rev. 28, 42-66.

Willamil, J., Badiola, I., Devillard, E, Geraert, P.A. \& Torrallardona, D., 2012. Wheat-barley-rye- or corn-fed growing pigs respond differently to dietary supplementation with a carbohydrase complex. J. Anim. Sci. 90, 824-832.

Woyengo, T.A., Beltranena, E. \& Zijlstra, R.T., 2014. Controlling feed cost by including alternative ingredients into pig diets: A review. J. Anim. Sci. 92, 1293-1305.

Yao, C.K., Muir, J.G. \& Gibson, P.R., 2016. Review article: Insights into colonic protein fermentation, its modulation and potential health implications. Aliment Pharmacol Ther. 43, 181-196.

Zervas, S. \& Zijlstra, R.T., 2002. Effects of dietary protein and fermentable fibre on nitrogen excretion patterns and plasma urea in grower pigs. J. Anim. Sci. 80, 3247-3256.

Zhu, C.L., Rademacher, M. \& de Lange, C.F.M., 2005. Increasing dietary pectin level reduces utilization of digestible threonine intake, but not lysine intake, or body protein deposition in growing pigs. J. Anim. Sci. 83, 1044-1053.

Zhu, C.L., Rademacher, M. \& de Lange, C.F.M., 2007. Intake of fermentable fibre and body protein deposition in pigs fed methionine or tryptophan limiting diets. EAAP Public. 124, 553-554.

Zijlstra, R.T. \& Beltranena, E., 2013. Swine convert co-products from food and biofuel industries into animal protein for food. Anim. Frontiers 3 (2), 48-53.

Zijlstra, R.T., Owusu-Asiedu, A. \& Simmins, P.H., 2010. Future of NSP-degrading enzymes to improve nutrient utilization of co-products and gut health in pigs. Livest. Sci. 134, 255-257. 